Method This is a pilot project where we emailed all the staff with different cultural background in one Hospital a Surfactant survey questionnaire seeking their opinion as a parent.

Results We received 151 responses of which 63\% were from females and $37 \%$ were from males. The majority of people who responded believe in Christianity (62\%), 24\% did not disclose there beliefs, $8 \%$ believe in Islam and $6 \%$ believe in Hinduism. More than half of the responses were from non-medical staff (55\%), doctors (23\%) and nursing staff (22\%). $74 \%$ who responded felt that the neonatal unit should stock all available types of surfactant and $79 \%$ responded that there should have been discussions regarding the different types of surfactant available and this should be included in antenatal counselling. Approximately $11 \%$ preferred either bovine or porcine surfactant based on religious beliefs, $36 \%$ preferred nonanimal derived surfactant which was for personal reasons and $53 \%$ had no preferences.

Conclusion In the current multicultural society, it is necessary to consider cultural beliefs of all patients. Paediatricians and Neonatologists must respect patient's and parent's autonomy and beliefs and they must be given sufficient information in a way that they can understand and are able to exercise their right to make informed decisions about their care. There are medico legal implications for ethical issues.

\section{PARMA PAP PROJECT: A READY TO USE THERAPEUTIC FOOD FOR MODERATELY MALNOURISHED CHILDREN IN SIERRA LEONE}

doi:10.1136/archdischild-2012-302724.1051

'M Verna, 'M Corradi, 'S Fantoni, 'I Proietti, 'F Savina, '2D Del Rio, 'R Virdis, 'M Vanelli, Post-graduate School of Paediatric, Human Nutrition Unit, Food Technology Laboratory, University of Parma, Parma, Italy. 'Pediatrics; ${ }^{2}$ Public Health, University of Parma, Parma, Italy

Background and Aims The Parma Pap Project started in 2008 as cooperation among Paediatric School, Human Nutrition Unit, Food Technology Laboratory, University of Parma and the NGO "Emergency", with the aim to develop a ready to use therapeutic food for the home-treatment of moderately malnourished African children. Parma Pap is a mix of peanuts, palm oil, milk powder and sugar, with high energy density and a micronutrient profile specifically designed on the base of WHO recommendations.

Methods The study included 330 moderately malnourished children between 6 months and 5 years, whose families referred to the Emergency Surgical Centre in Goderich, Sierra Leone. Patients with a Weight-for-Height Z score (WHZ) between 70\% and 80\% were enrolled and randomized into two groups: one received Feeding Program Supplementations (FPS: corn flour, palm oil and milk powder) + Parma Pap, whereas a control group received only FPS. The clinical follow-up lasted 13 weeks. Weight, height and WHZ were checked weekly and children were treated for concomitant infections.

Results The WHZ improvements were higher in children fed the Parma Pap with respect to the control group $(p<0.01$, MannWhitney U-test for independent samples). A detailed analysis of approximately 200 drop-outs was carried out to identify the most relevant factors involved.

Conclusions Providing Parma Pap in addition to standard feeding in malnourished children in Sierra Leone was an effective strategy to improve growth and in quickly replete WHZ. Information about participant drop-out will help increasing compliance in future feeding studies.

\section{PLANNING SURGERY IN CHILDREN WITH COMPLEX DISABILITY}

doi:10.1136/archdischild-2012-302724.1052
Y Akinsoji, A Jordan, R Ross Russell. Department of Paediatrics, Addenbrooke's Hospital, Cambridge, UK

Background Surgery in children with complex disability is ethically and practically difficult. Many disabled children benefit from relatively simple surgical procedures but the risks can deter some surgeons from performing the surgery. Some families do not fully understand the potential long term dependence that may follow.

Patients and Methodology A clinic appointment was made with a single consultant (RRR). A detailed history and examination was followed by a discussion of the risks of surgery. Specific risks discussed included an estimate of the risk of post-operative ventilation, and ventilation for longer than one week. Options for that eventuality (ranging from a tracheostomy and full intensive care through to palliative support) were then explained.

Results Twenty one visits in 20 patients have taken place. In five patients a Do Not Resuscitate order was already in place or had previously been discussed. Medical diagnoses were varied. Surgical procedures included gastrostomy formation, PEG change and muscle biopsy. Two children died before surgery and three others decided against the planned surgery in view of the risks. Of the 16 operations that took place, no children required post-operative ventilation. Length of stay varied between 1 day and 5 days (median 1.5 days). Nine patients have subsequently died.

Discussion Preoperative discussions were helpful to both families and staff, and helped clarify expectations. Extubation was often carried out with the parents present and agreement on nonreintubation. We suggest that brief surgical procedures in complex disabled children can be well tolerated and benefits from careful discussion ahead of surgery.

\section{NEONATAL ABSTINENCE SYNDROME: AN EVALUATION OF AN INFANT HOME-BASED WITHDRAWAL PROGRAM (IHBW)}

doi:10.1136/archdischild-2012-302724.1053

${ }^{1}$ C Smirk, 'E Bowman, 1.2LW Doyle, 'COF Kamlin. 'Royal Women's Hospital, Melbourne, Parkville; ${ }^{2}$ Department of Obstetrics, University of Melbourne, Melbourne, VIC, Australia

Background and Aims Conventional management of neonatal abstinence syndrome (NAS) often requires prolonged hospital admission, proving costly in both financial and social terms. However, safety issues often preclude the option of community-based withdrawal programs. We report the experience of our IHBW program.

Methods A retrospective audit was undertaken of infants older than 34 weeks gestation admitted to the Royal Women's Hospital Neonatal Unit for treatment of NAS between 2001-2010. Selected infants were offered the IHBW program from 2004.

Results 163 infants were admitted for treatment of NAS; 38 were managed on the IHBW program. $97 \%$ were exposed to opioids in utero. Morphine was used to treat 145 (89\%) infants with 18 (11\%) receiving either phenobarbitone or a combination of both.

Abstract 1053 Table 1 IHBW versus conventional management of NAS

\begin{tabular}{llll}
\hline & $\begin{array}{l}\text { Conventional } \\
\text { (N=125) }\end{array}$ & $\begin{array}{l}\text { IHBW } \\
\text { (N = 38) }\end{array}$ & $\begin{array}{l}\text { Mean difference } \\
\text { (95\% CI) or p value }\end{array}$ \\
\hline Maternal Age (yr) & $27.6(5.8)$ & $29.2(5.7)$ & $1.12(-1.0,3.2)$ \\
Gestational Age & $38.4(1.5)$ & $39.2(1.3)$ & $0.7(0.2,1.2)$ \\
Birth weight (g) & $2929(545)$ & $3087(355)$ & $158(-28,344)$ \\
NAS score before medication & $8.6(1.7)$ & $9.3(1.8)$ & $0.7(0.001,1.4)$ \\
Days of hospital stay & $39(12)$ & $19(12)$ & $-20.3(-24.7,-15.9)$ \\
Total dose morphine (mg/kg/birthweight) & $11.6(5.6)$ & $10.2(5.4)$ & $-1.4(-3.4,0.6)$ \\
Foster Care & $27(22 \%)$ & $2(5 \%)$ & 0.02 \\
Child Protection Involvement & $75(60 \%)$ & $9(24 \%)$ & $<0.001$ \\
Data in mean (SD) & & & \\
\hline
\end{tabular}

